\title{
Solitary Plasmacytoma of the Thoracic Spine
}

\section{Younus $\mathrm{A}^{1}$ and Kelly $\mathrm{A}^{*_{2}}$}

${ }^{1}$ FC Orthopedics (SA), Helen Joseph hospital; University of the Witwatersrand, Auckland Park, Johannesburg, South Africa

${ }^{2}$ MMed Neurosurgery, FC Neurosurgery (SA), George Mukhari Academic Hospital, Sefako Makgatho Health Sciences University, Molotlegi Street, Ga Rankuwa, Pretoria, South Africa

${ }^{*}$ Corresponding author: Kelly A, MMed Neurosurgery, FC Neurosurgery (SA), George Mukhari Academic Hospital, Sefako Makgatho Health Sciences University, Molotlegi Street, Ga Rankuwa, Pretoria, South Africa, 0204, Tel: +27 846773771, E-mail: adriankelly1000@yahoo.co.uk

Citation: Younus A, Kelly A (2018) Solitary Plasmacytoma of the Thoracic Spine. J Case Rep Stud 6(5): 501. doi: 10.15744/2348-9820.6.501

Received Date: August 09, 2018 Accepted Date: October 29, 2018 Published Date: October 31, 2018

\begin{abstract}
Background: Solitary bone plasmacytomas account for approximately $5 \%$ of plasma cell malignancies. These lesions are recognized as pre-malignant, if untreated, at least $50 \%$ will progress to multiple myeloma within 5 years of diagnosis. We present a case of a 54 year old male patient whom presented to our unit complaining of progressive axial backache complicated by acute onset paraplegia of 8 hours duration. Neurological examination revealed an incomplete L1 sensory level and no motor function of his lower limbs which had power $0 / 5$ in all dermatomes (Frankel B).

Results: The patient was immediately commenced on steroid therapy and an emergency surgical intervention was instituted whereby a trans-pleural approach was utilized to perform a T12 corpectomy and expandable cage reconstruction of the anterior and middle column of the T12 vertebrae. This was augmented by a lateral plate. The post-operative histology confirmed the lesion to be a plasmacytoma. Post-operatively significant improvement in the patient's neurology occurred whereby he improved to a point where he had normal sensation and slightly less than normal power in his lower limbs (Frankel D). He was referred for adjuvant radiotherapy. At his three month out-patient review the patient was ambulant with assistance and at his two year out-patient review he was free from recurrence.
\end{abstract}

Conclusion: We suggest that emergency surgical spinal cord decompression and reconstruction as a single stage procedure in the context of patients with solitary plasmacytomas with acute myelopathy can result in significant improvement. Expedience is the key to a successful outcome and in this case surgery was performed within 12 hours of onset of symptoms.

Keywords: Plasmacytoma Spine; Trans-Pleural Approach; Corpectomy

\section{Introduction}

Solitary bone plasmacytomas account for approximately $5 \%$ of plasma cell malignancies. These lesions are recognized as premalignant and, if untreated, at least $50 \%$ will progress to multiple myeloma within 5 years [1,2]. The primary goal of therapy is hence to arrest this progression and fortunately plasmacytomas do respond to moderate dose radiotherapy [3-5]. Local challenges at the site of the plasmacytoma do however need to be managed in their own right and with regards the thoracic spine indications for surgery include spinal instability, compressive symptoms either in the form of a radiculopathy or a myelopathy, and deformity.

\section{Materials and Methods}

A 54 year old male patient presented to our unit complaining of progressive axial backache complicated by acute onset paraplegia. Examination revealed incomplete neurology assessed to be a Frankel B where although sensation was intact with a L1 dermatomal sensory change, the patient had no motor function of his lower limbs. Imaging reveled gross spinal instability secondary to $>50 \%$ collapse of the T12 vertebral body and compression of the adjacent spinal cord. The lesion seen was expansive with significant osseous destruction localized to the involved vertebral body (Figure 1,2,3 and 4). The patient had a CT chest and abdomen as part of a metastatic screen which were all negative. His prostatic specific antigen was also within normal limits. The patient was urgently taken to surgery by the Neurosurgical-spine and Orthopedic-spine teams for a transthoracic trans-pleural approach where this anterior approach was utilized to undertake a corpectomy of the T12 vertebrae with reconstruction using an expandable cage with a lateral plate and autologous iliac bone graft (Figure 5 and 6). 


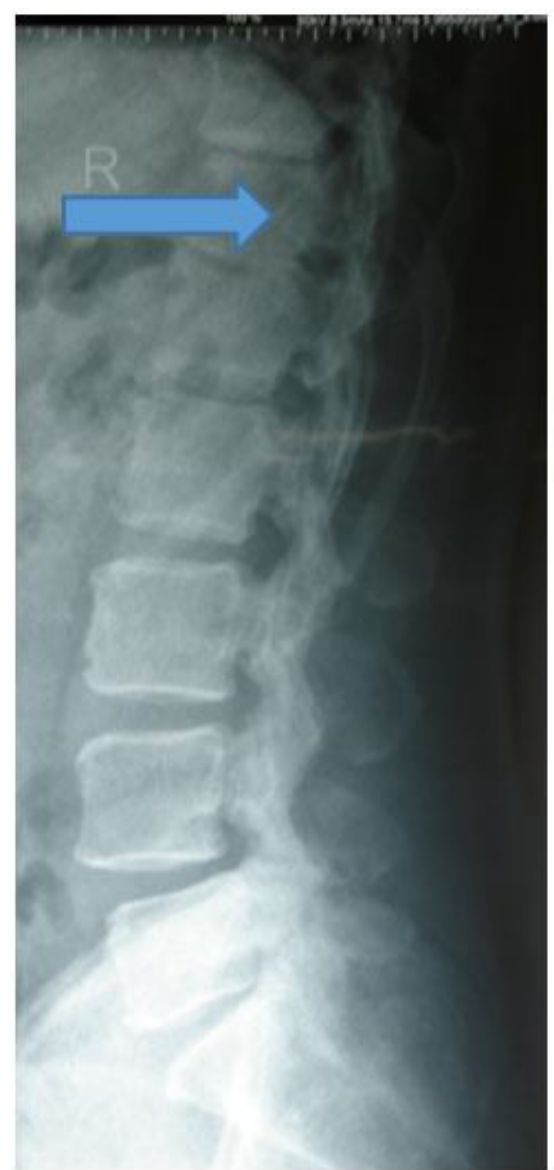

Figure 1: Pre-operative lateral lumbar spine X-ray lumbar; Demonstrating osteolysis of T12

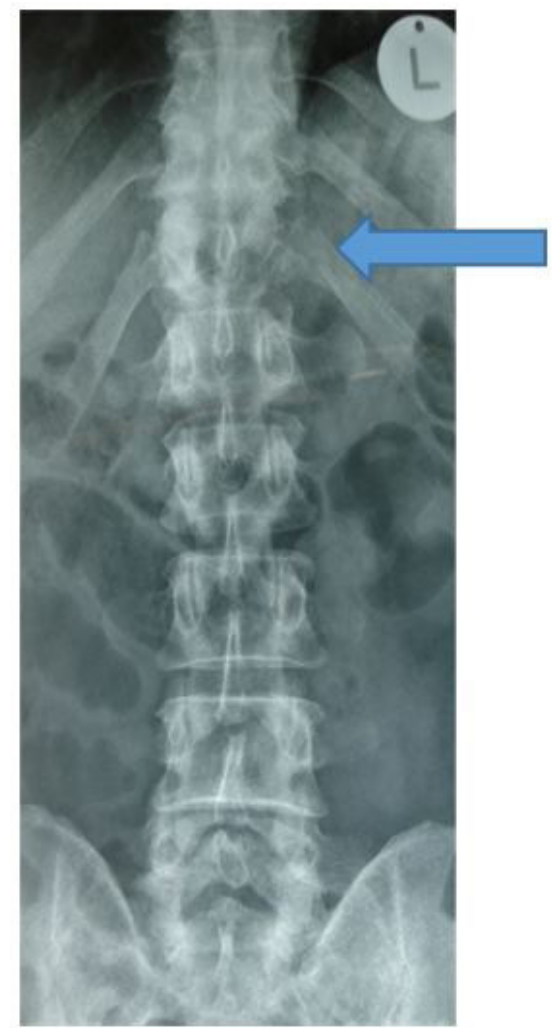

Figure 2: Pre-operative antero-posterior spine X-ray; Demonstrating osteolysis of pedicle of T12 


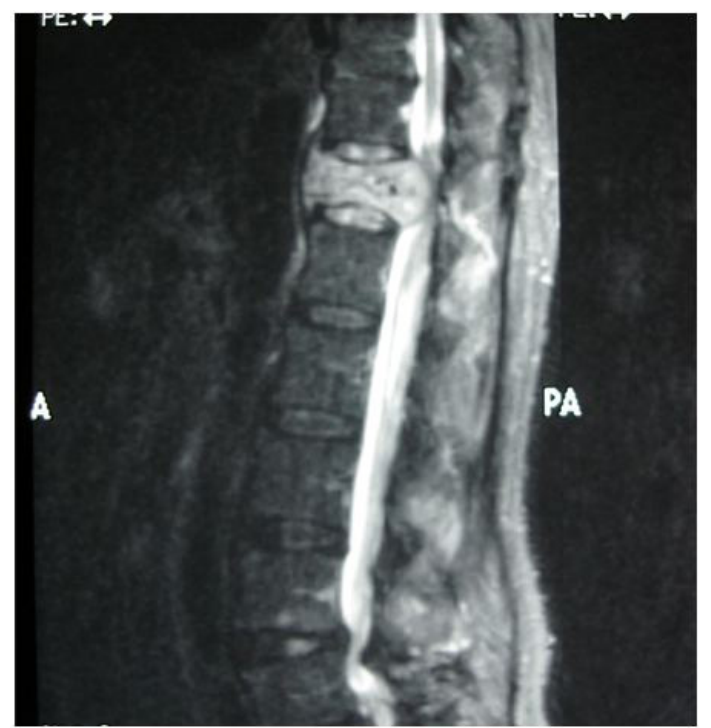

Figure 3: Pre-operative T2 sagittal MRI; Demonstrating collapse of T12 vertebral body with significant cord compression

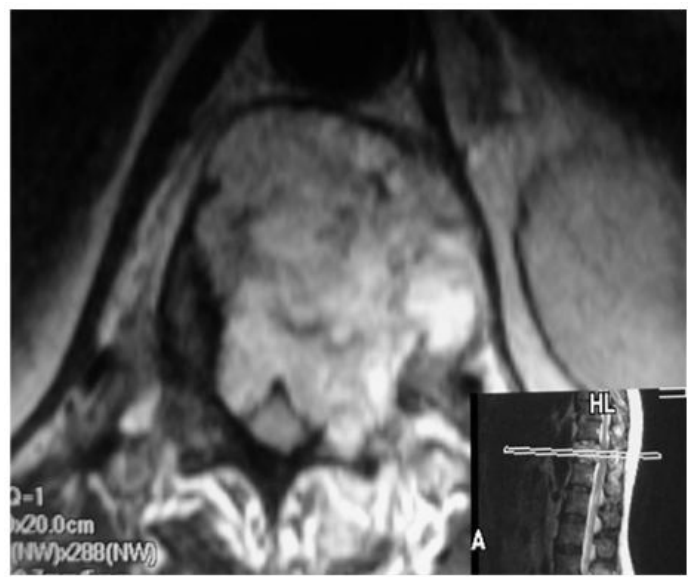

Figure 4: Pre-operative T2 axial MRI; Demonstrating expansile lesion within body of T12 and cord compression

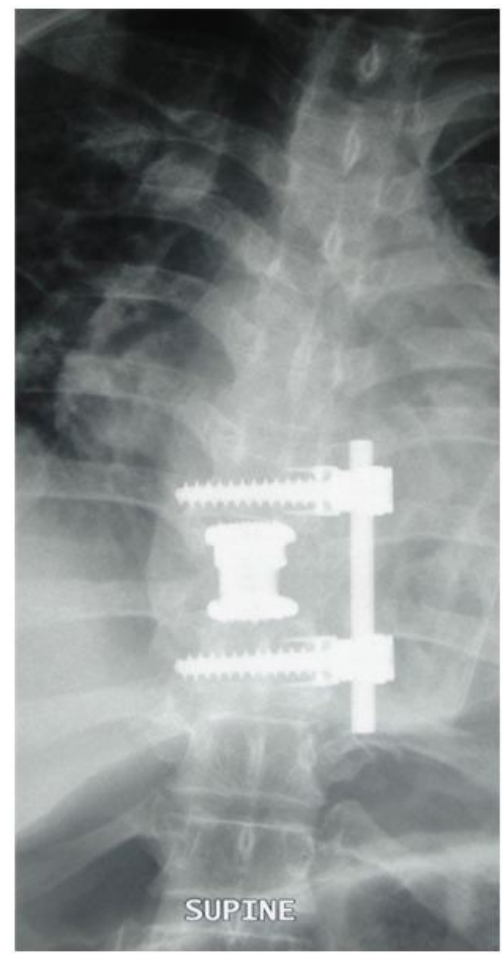

Figure 5: Post-operative thoracic spine antero-posterior X-ray; Demonstrating cage and lateral plate reconstruction post T12 corpectomy 


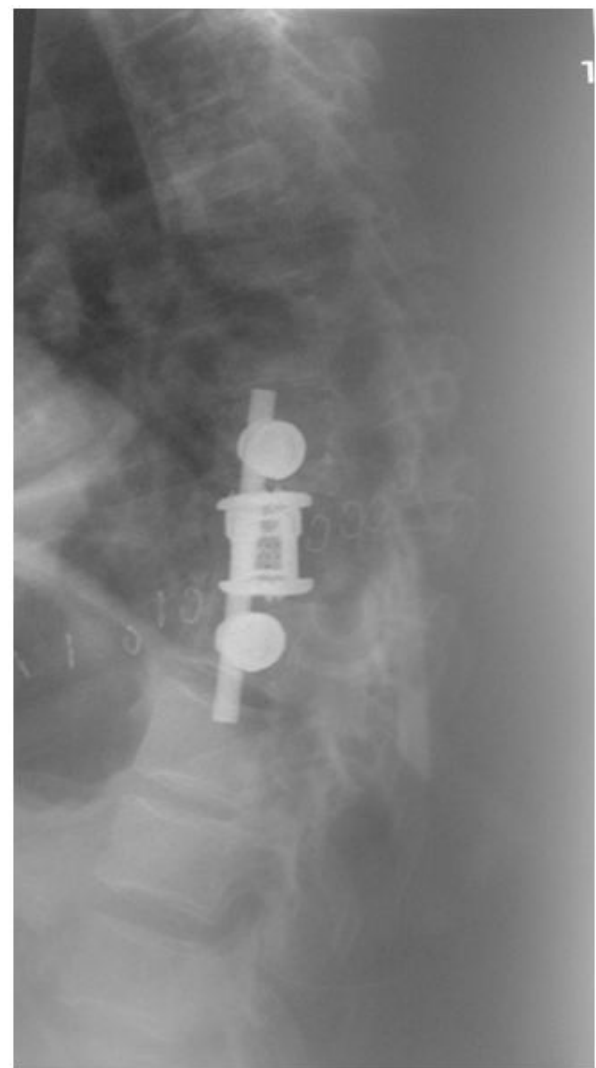

Figure 6: Post-operative thoracic spine lateral X-ray; Demonstrating cage and lateral plate reconstruction post T12 corpectomy

\section{Results}

Post operatively the patient improved to a Frankel D with preserved sensation and slightly less than normal power being demonstrated. At discharge the patient was ambulant with crutches. Histopathology confirmed the lesion to be a plasmacytoma and he subsequently received post-operative radiotherapy. The patient was seen in our out-patient department at two years postsurgery and is free of recurrence.

\section{Discussion}

A plasmacytoma is largely is largely regarded as a solitary form of multiple myeloma [6]. They occur in patients at a mean age of 55 years and are up to 2-3X more common in males [6,7]. This contrasts the demographics which characterize patients with multiple myeloma which occurs most commonly in the $6^{\text {th }}-7^{\text {th }}$ decade and has an equal gender distribution [8]. The plasmacytoma is however regarded as a precursor of multiple myeloma demanding effective management to avoid progression which occurs in over $50 \%$ of cases with 5 years [1,2]. Criteria for the diagnosis of a soft tissue plasmacytoma include the radiological lesion itself which must be confirmed to comprise a monoclonal plasma cell infiltrate. Essential negative findings that must accompany this positive finding are the absence of this infiltrate on bone marrow biopsy, absent serum and urine monoclonal and Benz jones proteins respectively on electrophoresis with no anemia, hypercalcemia nor renal impairment [3,9].

While plasmacytomas do respond well to moderate dose radiotherapy, a location in the spinal column presents unique challenges that dictate management in their own right [5]. These considerations are unique for the level involved but with regards the thoracic spine, which is the commonest site affected, spinal instability, compressive symptoms either in the form of a radiculopathy or a myelopathy, and deformity are regarded as indications for surgical decompression and stabilization [10].

Isolated anterior and middle column failure of the thoracic spine, as occurred in our case, is ideal for a transthoracic trans pleural approach as this avoids the disruption of the posterior tension band incurred by going posteriorly. By avoiding its disruption by not utilizing it as the surgical approach, the posterior column does not require a posterolateral instrumented fusion [11,12].

We report a successful outcome utilizing an anterior transthoracic Tran's pleural approach for a plasmacytoma of the T12 vertebral body. A patient underwent a T12 corpectomy with a cage and lateral plate reconstruction (Figure 4,5 and 6). This approach is suited for patients in whom the posterior column is intact [12]. 


\section{Conclusion}

Despite the devastating diagnosis of spinal instability secondary to collapse from a tumor this case demonstrates the benefit from expedient surgical management which in certain cases can even be curative in nature. These patients do however require close follow-up for the development of multiple myeloma and must be counseled to this effect.

\section{References}

1. Terada T (2011) Solitary plasmacytoma of the thoracic vertebra presenting with sudden paraplegia and back pain: A pathologic case report. Pathol Oncol Res 17: 167-9.

2. Yang JS, Cho YJ, Kang SH, Choi HJ (2013) Rapid progression of solitary plasmacytoma to multiple myeloma in lumbar vertebra. J Korean Neurosurg Soc 54: 426-30.

3. Bataille R, Sany J (1981) Solitary myeloma: clinical and prognostic features of a review of 114 cases. Cancer 48: 845-51.

4. Hong B, Hermann EJ, Reuter C, Brandis A, Krauss JK (2013) Outcome of surgical decompression of spinal mass lesions in non-Hodgeskins lymphoma and plasmacytoma. Clin Neurol Neurosurg 115: 2476-81.

5. Suh YG, Suh CO, Kim JS, Kim SJ, Pyun HO, et al. (2012) Radiotherapy for solitary plasmacytoma of bone and soft tissue. Ann Hematol 91: 1785-93.

6. Resnick D (2002) Diagnosis of Bone and Joint Disorders (4th Edn) Sauders; Phidelphia, PA, USA.

7. Guo SQ, Zhang L, Wang YF, Sun BC, Zhang LY, et al. (2013) Prognostic factors associated with solitary plasmacytoma. Onco Targets Ther 6: 1659-66.

8. Singh H, Meyer SA, Jenkins AL (2009) Treatment of primary vertebral tumors. Mount Sinai Journal of Medicine 76: 499-504.

9. Soutar R, Lucraft H, Jackson G, Reece A, Bird J, et al. (2004) Guidelines on the diagnosis and management of solitary plasmacytoma of bone and solitary extramedullary plasmacytoma. Br J Haematol 124: 717-26.

10. Hong B, Hermann EJ, Reuter C, Brandis A, Krauss JK (2013) Outcome of surgical decompression of spinal mass lesions in non-Hodgeskins lymphoma and plasmacytoma. Clin Neurol Neurosurg 115: 2476-81.

11. Arts MP, Bartels RH (2014) Anterior or posterior approach of thoracic disc herniation? A comparative cohort of mini transthoracic versus transpedicular discectomies. Spine J 14: 1654-62.

12. Mulier S, Debois V (1998) Thoracic disc herniations: transthoracic, lateral, or posterolateral approach? A review. Surg Neurol 49: 599-608. 